Historic, archived document

Do not assume content reflects current scientific knowledge, policies, or practices. 

A99.9 Fi64u

PLANT DEVELOPMENT AND YIELD ON MOUNTAIN GRASSLANDS IN SOUTHWESTERN MONTANA

U. S. DEPT. OF AGRICULTURE MATIONAL AGRICULTURALLIBRARY RECFIVED JUL $26 \quad 1977$ PRCR, EMENT SECTION
CURRENT SERIAL RECORDS
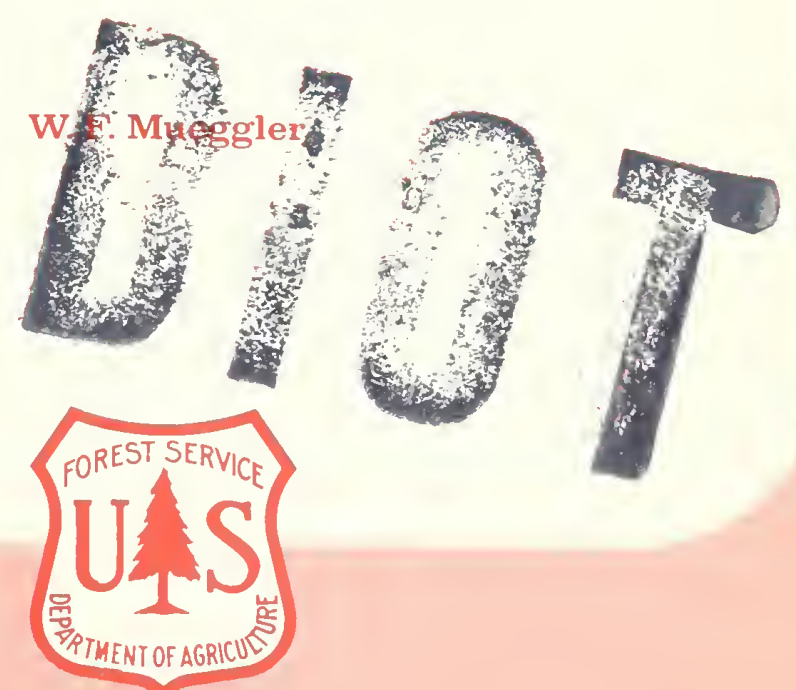

USDA Forest Serviee

Research Paper INT-124

May 1972

\section{$5111 \%$ \\ PLANT DEVELOPMENT \\ AND YIELD ON \\ MOUNTAIN GRASSLANDS \\ IN SOUTHWESTERN \\ MONTANA}

$p 1-20$

W. F. Mueggler

INTERMOUNTAIN FOREST AND RANGE EXPERIMENT STATION

Forest Service

U.S. Department of Agriculture

Ogden, Utah 84401

Robert W. Harris, Director 


\section{THE AUTHOR}

DR. WALTER F. MUEGGLER has been conducting range research for the Intermountain Station since 1949. Currently he is Project Leader of the Station's Mountain Grassland research work unit located in Bozeman, Montana. He holds graduate degrees in plant ecology from the University of Wisconsin and Duke University. 


\section{CONTENTS}

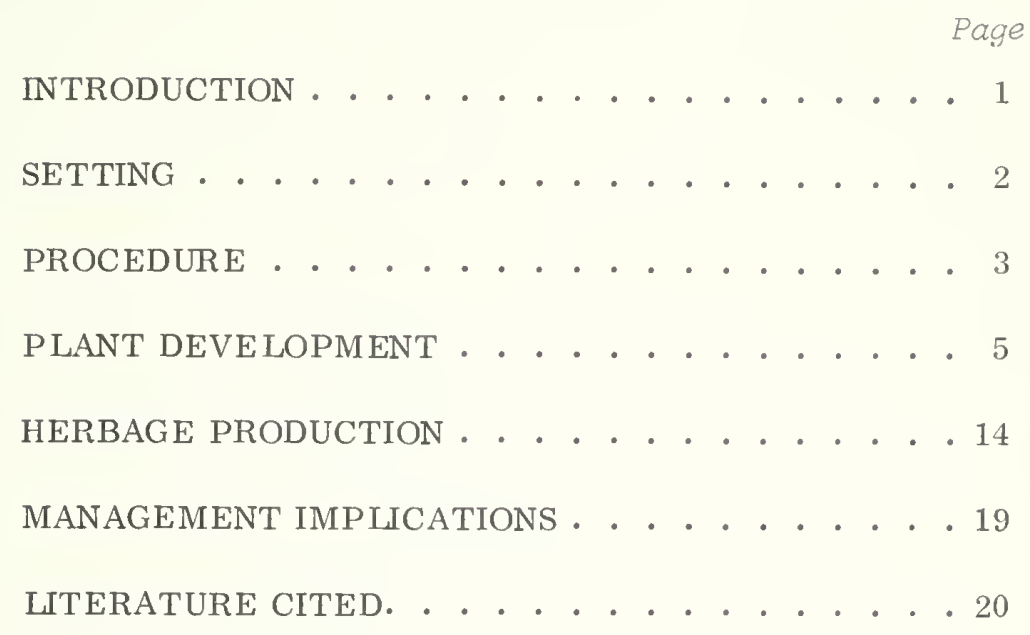

ABSTRACT

Plant development and herbage yields were observed for 5 consecutive years on natural mountain grasslands in southwestern Montana. Data were obtained from southwest and northeast exposures at elevations of 7,100 and 8,200 ft. Differences in development and production are compared between years, exposures, elevations, and species.

Beginning of growth in any 1 year differed between species and spread over about 3 weeks at the lower elevation and 2 weeks at the upper elevation. Lomatium was one of the earliest species to begin growth and Lupinus among the latest. Maximum difference in beginning of growth over the 5 years varied from as much as 32 days for Lomatium to as few as 13 days for Lupinus: the average for all species was 20 days. Again, depending upon the particular species, growth usually began 3 to 12 days later on northeast exposures than on southwest exposures and an average delay of about 1 week was observed for all species. Start of growth was usually 19 to 37 days later at the 8,200-ft. elevation than at 7,100 ft. and averaged 27 days later for all species.

The onset of flowering of different species in any 1 year was spread over about 10 weeks at the $7,100-\mathrm{ft}$. elevation and 7 weeks at the 8,200-ft. elevation. The greatest difference 
between years in onset of flowering was 22 days for early-blooming species and only 12 days for late-blooming species. Onset of flowering was generally about 1 week later on northeast than on southwest exposures and about 20 days later at 8,200 ft. than at 7,100 ft., although flowering time varies considerably between species.

The average time between cessation of flowering and start of seed dissemination was as short as 5 days for Delphinium and more than 40 days for Anemone, Besseya, and Fritillaria. Early-blooming species averaged 36 days and late-blooming species only 17 days. Generally, the grasses began seed dissemination between 18 and 20 days following flowering.

Drying times differed greatly between species. Generally, those that bloomed early dried earlier than those that bloomed later, but there were exceptions. The maximum difference between years in drying dates ranged from 19 days for the early-blooming forbs to 39 days for the grasses.

Average duration of the growth period at the 7,100-ft. elevation ranged from 62 days for Dodecatheon to 147 days for Danthonia. Differences between years in the duration of the growth period were as great as 28 days for the combined forbs and 43 days for the combined grasses. The average duration of the growth period for species common to both elevations was 103 days at 7,100 ft. and 94 days at $8,200 \mathrm{ft}$.

Herbage production on southwest exposures was generally less than that on northeast exposures, averaging 58 percent of that on the northeast exposure at the 7,100-ft. elevation and 90 percent at $8,200 \mathrm{ft}$. The relative proportions of graminoid to forb production was inconsistent between both exposures and elevations.

Yearly variations in herbage production were almost twice as great on northeast as they were on southwest exposures. Production of forbs varied more than that of graminoids. Relative variations in production for individual species were usually greater than for vegetation classes and totals.

A positive correlation existed betwen May precipitation and herbage production. A negative correlation existed between maximum temperatures of the shaded soil surface in June and July and herbage production. A multiple regression equation, utilizing a combination of five weather measurements, was developed that predicts total herbage production with an $\mathrm{R}^{2}$ of 0.86 . Similar equations, utilizing different combinations of weather measurements, were developed that predict total yield of forbs having an $\mathrm{R}^{2}$ of 0.74 and total yield of graminoids having an $\mathrm{R}^{2}$ of 0.52 . 


\section{INTRODUCTION}

Annual differences in plant growth and production are among the most commonly observed phenomena on our native rangelands. In addition, knowledge of growth and production are fundamental to understanding the dynamics of the grassland ecosystem. Despite their conspicuousness and importance, these phenomena have not been documented for all range types. Mountain grasslands of western Montana are among those areas where information is generally lacking on yearly variations in plant development and herbage yield.

During a continuing study of the relation between plant growth and weather, I accumulated data on variations in development and yield at four different mountain grass1 and sites. A summary of these data is presented here for those interested in mountain grassland vegetation. Resource managers should find this summary useful for judging the amount of year-to-year variability in range readiness for grazing and the variability in grazing capacity that can be expected to occur naturally on such areas. 


\section{SETTING}

The four study areas selected were near the north end of the Gravelly Range in southwestern Montana, approximately 12 miles southeast of Virginia City. The areas were on gentle southwest and northeast exposures, two at an elevation of 7,100 feet and two at 8,200 feet. The two lower areas were within 200 yards of each other, whereas the higher areas, which were 5 miles away, were within 700 yards of each other. This arrangement permitted determination of differences in development and production between extreme exposures and between elevations.

The soils, derived principally from limestone, were generally deeper at the upper elevation and on northeast exposures. Annual precipitation usually ranged between 20 and 30 inches; about half fell from May through October. Precipitation during May and June was usually heavy; it accounted for about one-fourth of the yearly total.

All four areas were natural mountain grasslands dominated by Festuca idahoensis. Forbs were abundant. Many graminoid and forb species were common to all four areas, but other species were restricted to specific sites.

I collected data on plant development and herbage yields on all study sites for 5 consecutive years, 1964-1968. Each area was adjacent to a weather station established to measure the climate on mountain grasslands. I recently described weather variations over the same 5-year period (Mueggler 1971). 


\section{PROCEDURE}

Seasonal development was recorded for plant species common to all four areas and for conspicuous species on each area. Individual plants of selected species were marked for repeated observation. Exclosures protected these plants from livestock. Dates of various developmental stages for each species were established from the development of marked plants plus supplemental observation of the surrounding plants. If development of a marked plant appeared to be abnormal, a more typical specimen was selected for subsequent observations. The areas were visited weekly from the onset of active growth in the spring until the majority of the species became dormant in the fall.

The developmental stages discussed in this report are as follows:

Forbs

1. Growth starts--first evidence of current year's herbaceous growth.

2. First bloom--first fully developed blossoms.

3. Full bloom--aspect of most individuals of the species in full flower.

4. Bloom over--approximately 90 percent of flowering completed.

5. Dissemination starts--first evidence of seed drop.

6. Plant dried--at least 90 percent of the herbage dried.

Grasses

1. Growth starts--first evidence of current year's herbaceous growth.

2. Flower stalks appear--first evidence of booted flowering culms.

3. Flowers in bloom--approximately 25 percent or more of flowers in anthesis.

4. Dissemination starts--first evidence of seed drop.

5. Drying--at least 10 percent of herbage dried.

6. Plant dried--at least 90 percent of the herbage dried. 
Herbage production was measured on each area from 50 permanent 4.8 -ft. ${ }^{2}$ plots. These plots were distributed in a series of 10 sets, five plots per set, over a relatively uniform portion of the grassland. Production was measured each year when the vegetation appeared to have reached full growth and just before many species began to dry. Generally, production data were taken between the middle and end of July at the lower elevation and about 3 weeks later at the upper elevation.

Production was determined by combining percent-weight estimates and clipping. This technique is similar, but not identical, to that proposed by Hutchings and Schmautz (1969); it is based on the concept that it is more efficient to estimate yields as a percent of total yield rather than directly as weight units. Each year, one plot in each set of five was protected from livestock grazing by a wire cage. This plot was considered the reference plot for that particular year. The reference plot was changed each year; thus, a plot served as a reference only once in 5 years. Total herbage production on each of the remaining four plots in the set was estimated as a percent of the total production on the set's reference plot. These estimates were adjusted to compensate for current livestock utilization, which usually was minor. The weight of each species on a plot was then estimated as a percent of the total production on the plot; these estimates were also adjusted to compensate for utilization. The vegetation on the reference plot was then clipped to ground level. Individual production of four selected species served as a continual check on the accuracy of the percent-weight estimates. The total production on the reference plot was used as a basis for estimating production on other plots in the set. Then, the green weight of individual

species on each plot was computed. Later, the clipped material was dried to obtain the percent dry matter by important species. All production estimates were converted to dry weights expressed in pounds per acre of air-dry material. 


\section{PLANT DEVELOPMENT}

The average dates of various developmental stages for 23 forbs and five grasses growing on the southwest exposure at 7,100 ft. elevation are shown in figures 1 and 2 . The beginning of annual herbaceous growth for the 23 forbs spread over more than 3 weeks. Lomatium generally was the first plant to begin growth (April 27) and Lupinus the last (May 22). Usually, all five of the grasses studied began to grow during the first week in May.

The greatest difference between years for the beginning of growth was as much as 32 days for Linum and as few as 13 days for Lupinus (table 1). For most species this difference was from 16 to 24 days, and averaged 20 days (tables 1 and 2). In other words, the general start of plant growth varied approximately 3 weeks in just one 5-year period. A much greater yearly variation (45 days for grasses and 50 days for forbs) was recorded by Costello and Price (1939) over a 10-year period on mountain herblands in Utah.

The onset of flowering of the 23 forbs spread over more than 10 weeks (fig. 1). These forbs can be categorized according to when they reach full bloom as early, middle, or late season bloomers. The early bloomers are the first six species that generally reach full bloom sometime in May. The next six species, the middle season bloomers, reach full bloom in June, and the remaining species, the late season bloomers, usually do not reach full flower until sometime in July. The flowers of Lomatium, Anemone, Besseya, and Fritizlaria are among the first signs that spring has finally arrived on these mountain grasslands, while the flowers of Achizlea, Gaizzardia, and Campanuza are a warning that the annual floral display will soon end. The grasses ordinarily flower in mid-July; approximately 1 week elapses between the onset of flowering in the earliest, Danthonia, and the latest, Agropyron (fig. 2). 


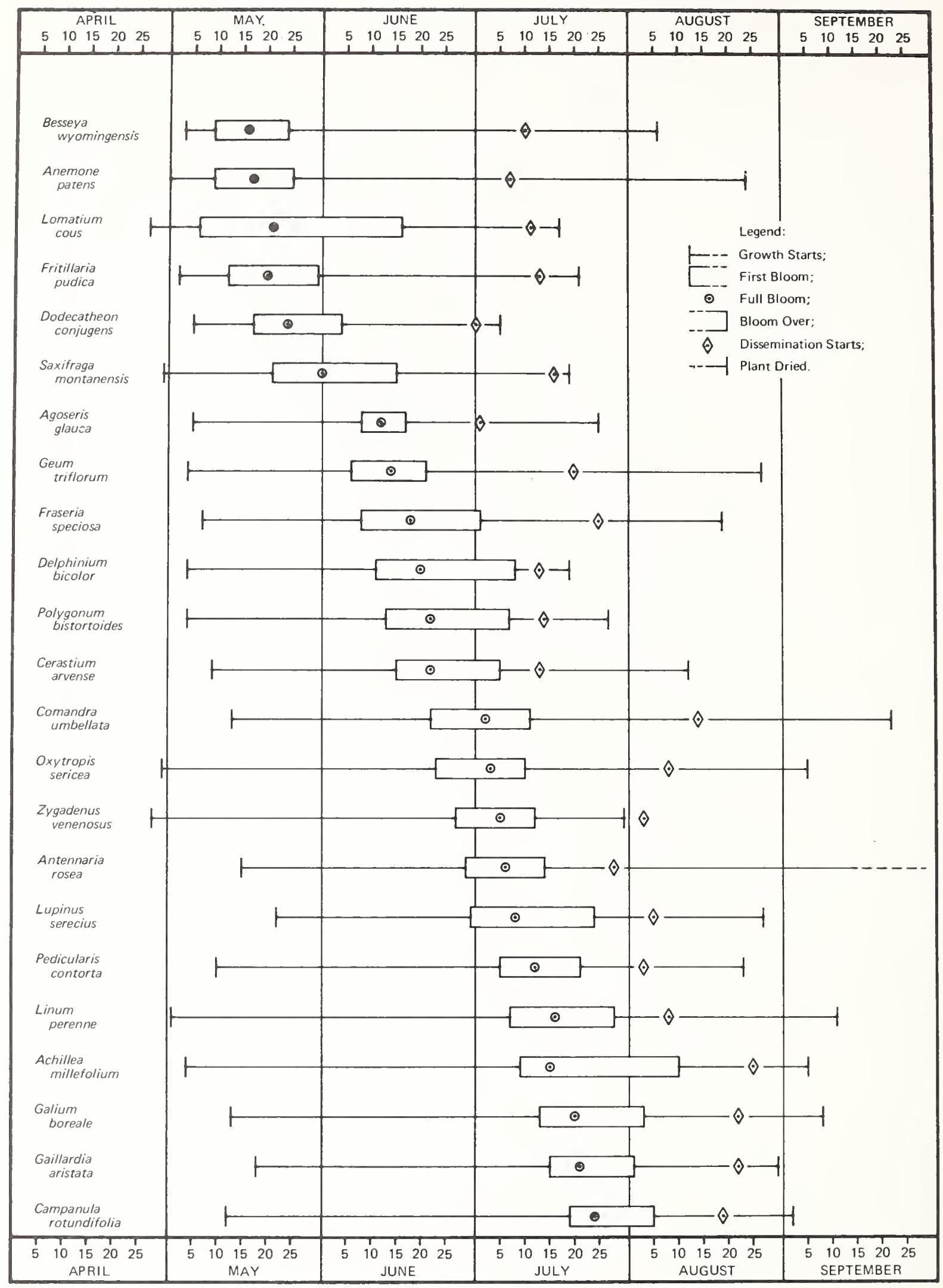

Figure 1.--The 5-year mean of developmental events for forbs on a southwest exposure at 7,100 ft.-elevation. 


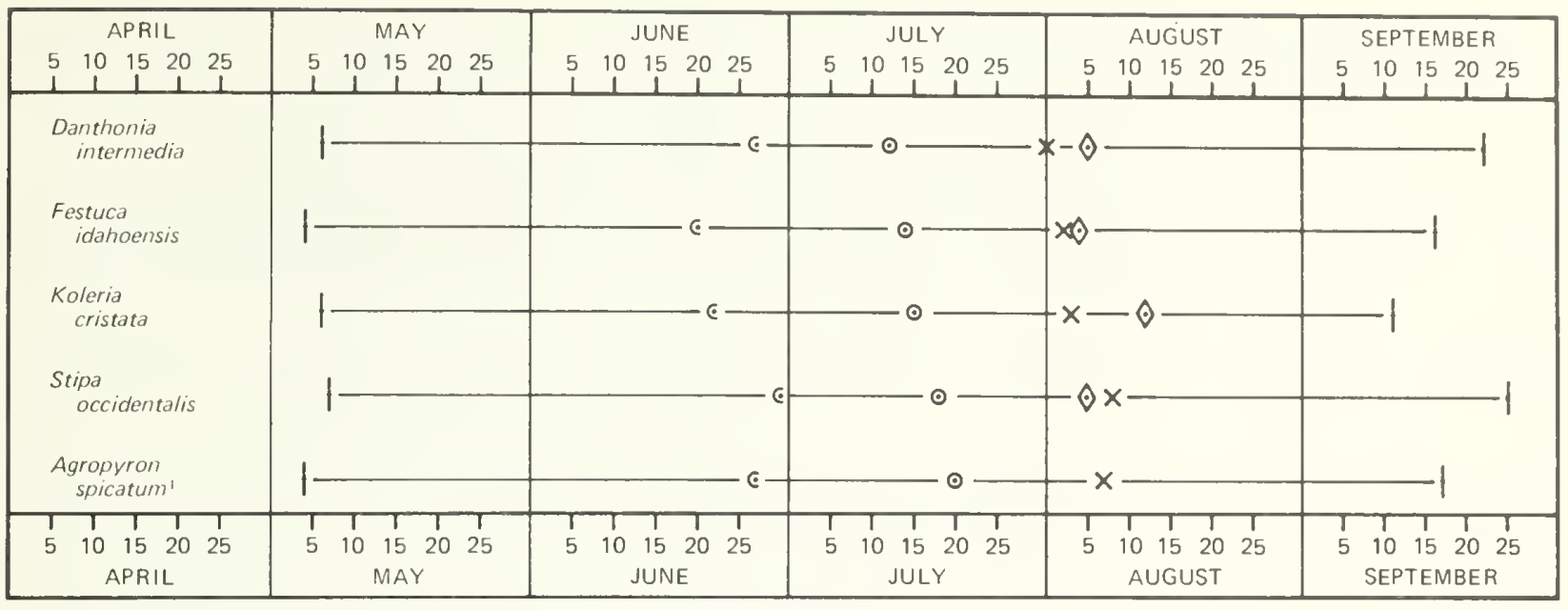

${ }^{1}$ No seed produced during 5 year period because of heavy insect infestation.

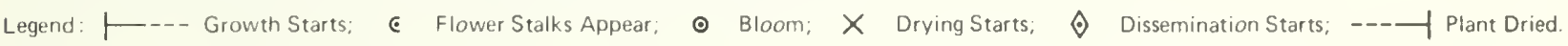

Figure 2.--The 5-year mean of developmental events for grasses

on a southwest exposure at ?,100-ft. elevation.

The maximum difference between years in time of first bloom for the six earlyflowering forbs ranged from 18 to 33 days (average was 22 days). This difference was about the same for the six forbs that bloom in midseason. However, the maximum difference between years in time of first bloom of the 11 late-flowering forbs ranged from 8 to 21 days and averaged only 12 days. The difference between the earliest and latest dates of full bloom corresponded closely to that for first bloom for all three groups of species. Apparently, temperature is a dominant factor in control of flowering of species that bloom early or in midseason. Retarding effects of a late spring and accelerating effects of an early spring are still effective in early June when the midseason plants begin to flower, but have little effect by the time the late-blooming species start to flower in late June and early July. The reduced yearly variation in date of flowering of the late-blooming species could reflect this group's greater dependence on photoperiod than on temperature control of flowering.

The greatest difference between years in the date of flower stalk appearance for the grasses ranged from 10 days for Danthonia to 28 days for Festuca; it averaged 17 days for all five species studied (table 2). Yearly differences were reduced to an average of 11 days ( 6 days for Agropyron to 19 days for Danthonia) by the time these grasses flowered. Interestingly, the date of blooming for Festuca varied relatively little between years ( 8 days), and Danthonia varied greatly (19 days); this was just the opposite of the variation that existed when the flower stalks first appeared. Average elapsed time between first appearance of flower stalks and blooming was 14 days for Danthonia, 18 days for Stipa, and approximately 23 days for Agropyron, Festuca, and Koeleria.

The duration of flowering (fig. 1) denotes the time each species of forb had a floral aspect. The precise date of flowering differed between individual plants of the same species; this difference was much greater in some species than in others.

Lomatium and Achillea prolonged their floral aspect over at least 4 weeks, whereas that of most species usually lasted 2 to 3 weeks. 
Table 1.--Earliest (E), latest $(L)$, and mean $(M)$ dates of developmental events for forbs on the southwest exposure at 7,100-ft. elevation over a 5-year period.

\begin{tabular}{|c|c|c|c|c|c|c|c|}
\hline Species & : Code & $\begin{array}{l}: \text { Active } \\
: \text { growth } \\
: \text { starts } \\
\end{array}$ & $\begin{array}{l}: \text { Flower- } \\
: \text { ing } \\
: \text { starts }\end{array}$ & $\begin{array}{ll}: & \\
: & \text { Full } \\
: & \text { bloom } \\
\end{array}$ & $\begin{array}{l}: \text { Flower- } \\
: \text { ing } \\
: \text { over } \\
\end{array}$ & $\begin{array}{l}: \text { Seed dis- } \\
: \text { semination } \\
: \quad \text { starts } \\
\end{array}$ & $\begin{array}{ll}: & \\
: & \text { P1ant } \\
: & \text { dried } \\
\end{array}$ \\
\hline $\begin{array}{l}\text { Achizrea } \\
\text { mizzeforium }\end{array}$ & $\begin{array}{l}E \\
L \\
M\end{array}$ & $\begin{array}{l}4 / 28 \\
5 / 12 \\
5 / 4\end{array}$ & $\begin{array}{l}6 / 28 \\
7 / 15 \\
7 / 9\end{array}$ & $\begin{array}{l}7 / 6 \\
7 / 21 \\
7 / 15\end{array}$ & $\begin{array}{l}7 / 30 \\
8 / 31 \\
8 / 10\end{array}$ & $\begin{array}{l}8 / 11 \\
9 / 4 \\
8 / 25\end{array}$ & $\begin{array}{l}8 / 21 \\
9 / 27 \\
9 / 5\end{array}$ \\
\hline $\begin{array}{l}\text { Agoseris } \\
\text { grauca }\end{array}$ & $\begin{array}{l}E \\
L \\
M\end{array}$ & $\begin{array}{l}4 / 28 \\
5 / 15 \\
5 / 5\end{array}$ & $\begin{array}{l}5 / 22 \\
7 / 7 \\
6 / 8\end{array}$ & $\begin{array}{l}5 / 26 \\
7 / 11 \\
6 / 12\end{array}$ & $\begin{array}{l}6 / 1 \\
7 / 17 \\
6 / 17\end{array}$ & $\begin{array}{l}6 / 15 \\
7 / 19 \\
7 / 1\end{array}$ & $\begin{array}{l}7 / 9 \\
8 / 16 \\
7 / 25\end{array}$ \\
\hline $\begin{array}{l}\text { Anemone } \\
\text { patens }\end{array}$ & $\begin{array}{l}E \\
L \\
M\end{array}$ & $\begin{array}{l}4 / 18 \\
5 / 10 \\
4 / 31\end{array}$ & $\begin{array}{l}4 / 30 \\
5 / 18 \\
5 / 9\end{array}$ & $\begin{array}{l}5 / 9 \\
5 / 24 \\
5 / 17\end{array}$ & $\begin{array}{l}5 / 18 \\
6 / 2 \\
5 / 25\end{array}$ & $\begin{array}{l}6 / 25 \\
7 / 16 \\
7 / 7\end{array}$ & $\begin{array}{l}8 / 20 \\
8 / 30 \\
8 / 24\end{array}$ \\
\hline $\begin{array}{l}\text { Antennaria } \\
\text { rosea }\end{array}$ & $\begin{array}{l}E \\
L \\
M\end{array}$ & $\begin{array}{l}5 / 3 \\
5 / 26 \\
5 / 15\end{array}$ & $\begin{array}{l}6 / 18 \\
7 / 6 \\
6 / 29\end{array}$ & $\begin{array}{l}6 / 24 \\
7 / 11 \\
7 / 6\end{array}$ & $\begin{array}{l}7 / 8 \\
7 / 17 \\
7 / 14\end{array}$ & $\begin{array}{l}7 / 24 \\
8 / 5 \\
7 / 28\end{array}$ & $\begin{array}{l}-- \\
-- \\
--\end{array}$ \\
\hline $\begin{array}{l}\text { Besseya } \\
\text { wyomingensis }\end{array}$ & $\begin{array}{l}E \\
L \\
M\end{array}$ & $\begin{array}{l}4 / 22 \\
5 / 10 \\
5 / 3\end{array}$ & $\begin{array}{l}4 / 30 \\
5 / 18 \\
5 / 9\end{array}$ & $\begin{array}{l}5 / 4 \\
5 / 26 \\
5 / 16\end{array}$ & $\begin{array}{l}5 / 8 \\
6 / 2 \\
5 / 24\end{array}$ & $\begin{array}{l}6 / 30 \\
7 / 20 \\
7 / 10\end{array}$ & $\begin{array}{l}7 / 30 \\
8 / 10 \\
8 / 6\end{array}$ \\
\hline $\begin{array}{l}\text { Campanuza } \\
\text { rotundifolia }\end{array}$ & $\begin{array}{l}E \\
L \\
M\end{array}$ & $\begin{array}{l}5 / 1 \\
5 / 20 \\
5 / 12\end{array}$ & $\begin{array}{l}7 / 15 \\
7 / 24 \\
7 / 19\end{array}$ & $\begin{array}{l}7 / 20 \\
8 / 1 \\
7 / 24\end{array}$ & $\begin{array}{l}7 / 28 \\
8 / 13 \\
8 / 5\end{array}$ & $\begin{array}{l}8 / 13 \\
8 / 28 \\
8 / 19\end{array}$ & $\begin{array}{l}8 / 21 \\
9 / 17 \\
9 / 2\end{array}$ \\
\hline $\begin{array}{l}\text { Cerastium } \\
\text { arvense }\end{array}$ & $\begin{array}{l}E \\
L \\
M\end{array}$ & $\begin{array}{l}5 / 1 \\
5 / 20 \\
5 / 9\end{array}$ & $\begin{array}{l}5 / 28 \\
6 / 27 \\
6 / 15\end{array}$ & $\begin{array}{l}6 / 8 \\
7 / 1 \\
6 / 22\end{array}$ & $\begin{array}{l}6 / 28 \\
7 / 12 \\
7 / 5\end{array}$ & $\begin{array}{l}7 / 3 \\
7 / 20 \\
7 / 13\end{array}$ & $\begin{array}{l}7 / 30 \\
9 / 5 \\
8 / 12\end{array}$ \\
\hline $\begin{array}{l}\text { Comandra } \\
\text { umberzata }\end{array}$ & $\begin{array}{l}E \\
L \\
M\end{array}$ & $\begin{array}{l}5 / 1 \\
5 / 25 \\
5 / 13\end{array}$ & $\begin{array}{l}6 / 19 \\
6 / 27 \\
6 / 22\end{array}$ & $\begin{array}{l}6 / 28 \\
7 / 5 \\
7 / 2\end{array}$ & $\begin{array}{l}7 / 2 \\
7 / 18 \\
7 / 11\end{array}$ & $\begin{array}{l}\left({ }^{l}\right) \\
8 / 14\end{array}$ & $\begin{array}{c}9 / 6 \\
10 / 10 \\
9 / 22\end{array}$ \\
\hline $\begin{array}{c}\text { Dezphinium } \\
\text { bicolor }\end{array}$ & $\begin{array}{l}E \\
L \\
M\end{array}$ & $\begin{array}{l}4 / 21 \\
5 / 17 \\
5 / 4\end{array}$ & $\begin{array}{l}5 / 22 \\
6 / 26 \\
6 / 11\end{array}$ & $\begin{array}{l}6 / 6 \\
7 / 1 \\
6 / 20\end{array}$ & $\begin{array}{l}6 / 28 \\
7 / 18 \\
7 / 8\end{array}$ & $\begin{array}{l}7 / 2 \\
7 / 21 \\
7 / 13\end{array}$ & $\begin{array}{l}7 / 8 \\
7 / 26 \\
7 / 19\end{array}$ \\
\hline $\begin{array}{l}\text { Dodecatheon } \\
\text { conjugens }\end{array}$ & $\begin{array}{l}E \\
L \\
M\end{array}$ & $\begin{array}{l}4 / 28 \\
5 / 14 \\
5 / 5\end{array}$ & $\begin{array}{l}5 / 5 \\
5 / 27 \\
5 / 17\end{array}$ & $\begin{array}{l}5 / 11 \\
6 / 3 \\
5 / 24\end{array}$ & $\begin{array}{l}5 / 15 \\
6 / 15 \\
6 / 4\end{array}$ & $\begin{array}{l}\left({ }^{1}\right) \\
6 / 4\end{array}$ & $\begin{array}{l}6 / 20 \\
7 / 13 \\
7 / 5\end{array}$ \\
\hline $\begin{array}{l}\text { Frasera } \\
\quad \text { speciosa }\end{array}$ & $\begin{array}{l}E \\
L \\
M\end{array}$ & $\begin{array}{l}4 / 26 \\
5 / 16 \\
5 / 7\end{array}$ & $\begin{array}{l}5 / 25 \\
6 / 18 \\
6 / 8\end{array}$ & $\begin{array}{l}6 / 3 \\
6 / 28 \\
6 / 18\end{array}$ & $\begin{array}{l}6 / 23 \\
7 / 6 \\
7 / 1\end{array}$ & $\begin{array}{l}7 / 15 \\
8 / 1 \\
7 / 25\end{array}$ & $\begin{array}{l}7 / 30 \\
9 / 2 \\
8 / 19\end{array}$ \\
\hline $\begin{array}{c}\text { Fritizzaria } \\
\text { pudica }\end{array}$ & $\begin{array}{l}E \\
L \\
M\end{array}$ & $\begin{array}{l}4 / 20 \\
5 / 13 \\
5 / 2\end{array}$ & $\begin{array}{l}5 / 2 \\
5 / 25 \\
5 / 12\end{array}$ & $\begin{array}{l}5 / 10 \\
5 / 30 \\
5 / 20\end{array}$ & $\begin{array}{l}5 / 18 \\
6 / 10 \\
5 / 20\end{array}$ & $\begin{array}{l}7 / 19 \\
7 / 27 \\
7 / 13\end{array}$ & $\begin{array}{l}6 / 25 \\
8 / 5 \\
7 / 21\end{array}$ \\
\hline
\end{tabular}


Table 1.--(con.)

\begin{tabular}{|c|c|c|c|c|c|c|c|}
\hline Species & $:$ Code & $\begin{array}{l}: \text { Active } \\
: \text { growth } \\
: \text { starts } \\
\end{array}$ & $\begin{array}{l}: \text { Flower- } \\
: \text { ing } \\
: \text { starts } \\
\end{array}$ & $\begin{array}{ll}: & \\
: & \text { Full } \\
: & \text { bloom }\end{array}$ & $\begin{array}{l}: \text { Flower- } \\
: \text { ing } \\
: \text { over }\end{array}$ & $\begin{array}{l}: \text { Seed dis- } \\
: \text { semination } \\
: \text { starts }\end{array}$ & $\begin{array}{ll}: & \text { Plant } \\
: & \text { dried } \\
\end{array}$ \\
\hline $\begin{array}{l}\text { Galium } \\
\text { boreale }\end{array}$ & $\begin{array}{l}E \\
L \\
M\end{array}$ & $\begin{array}{l}5 / 3 \\
5 / 24 \\
5 / 13\end{array}$ & $\begin{array}{l}7 / 18 \\
7 / 18 \\
7 / 13\end{array}$ & $\begin{array}{l}7 / 14 \\
7 / 25 \\
7 / 20\end{array}$ & $\begin{array}{l}7 / 27 \\
8 / 14 \\
8 / 3\end{array}$ & $\begin{array}{l}8 / 16 \\
9 / 8 \\
8 / 22\end{array}$ & $\begin{array}{l}8 / 27 \\
9 / 18 \\
9 / 8\end{array}$ \\
\hline $\begin{array}{r}\text { Gailzardia } \\
\text { aristata }\end{array}$ & $\begin{array}{l}E \\
L \\
M\end{array}$ & $\begin{array}{l}5 / 7 \\
5 / 28 \\
5 / 18\end{array}$ & $\begin{array}{l}7 / 11 \\
7 / 22 \\
7 / 15\end{array}$ & $\begin{array}{l}7 / 16 \\
7 / 25 \\
7 / 21\end{array}$ & $\begin{array}{l}7 / 18 \\
8 / 12 \\
8 / 1\end{array}$ & $\begin{array}{l}8 / 11 \\
9 / 3 \\
8 / 22\end{array}$ & $\begin{array}{l}8 / 20 \\
9 / 10 \\
8 / 30\end{array}$ \\
\hline $\begin{array}{l}\text { Geum } \\
\text { triftorum }\end{array}$ & $\begin{array}{l}E \\
L \\
M\end{array}$ & $\begin{array}{l}4 / 26 \\
5 / 12 \\
5 / 4\end{array}$ & $\begin{array}{l}5 / 29 \\
6 / 15 \\
6 / 6\end{array}$ & $\begin{array}{l}6 / 6 \\
6 / 20 \\
6 / 14\end{array}$ & $\begin{array}{l}6 / 10 \\
6 / 30 \\
6 / 21\end{array}$ & $\begin{array}{l}7 / 11 \\
7 / 25 \\
7 / 20\end{array}$ & $\begin{array}{l}8 / 16 \\
9 / 13 \\
8 / 27\end{array}$ \\
\hline $\begin{array}{l}\text { Linum } \\
\text { perenne }\end{array}$ & $\begin{array}{l}E \\
L \\
M\end{array}$ & $\begin{array}{l}4 / 21 \\
5 / 22 \\
5 / 1\end{array}$ & $\begin{array}{l}7 / 1 \\
7 / 12 \\
7 / 7\end{array}$ & $\begin{array}{l}7 / 8 \\
7 / 25 \\
7 / 16\end{array}$ & $\begin{array}{l}7 / 18 \\
8 / 5 \\
7 / 28\end{array}$ & $\begin{array}{l}7 / 22 \\
8 / 18 \\
8 / 8\end{array}$ & $\begin{array}{l}8 / 26 \\
9 / 24 \\
9 / 11\end{array}$ \\
\hline $\begin{array}{l}\text { Lomatium } \\
\text { cous }\end{array}$ & $\begin{array}{l}\mathrm{E} \\
\mathrm{L} \\
\mathrm{M}\end{array}$ & $\begin{array}{l}4 / 18 \\
5 / 10 \\
4 / 27\end{array}$ & $\begin{array}{l}4 / 20 \\
5 / 23 \\
5 / 6\end{array}$ & $\begin{array}{l}5 / 12 \\
5 / 30 \\
5 / 21\end{array}$ & $\begin{array}{l}6 / 6 \\
6 / 27 \\
6 / 16\end{array}$ & $\begin{array}{l}7 / 7 \\
7 / 21 \\
7 / 11\end{array}$ & $\begin{array}{l}7 / 10 \\
7 / 26 \\
7 / 17\end{array}$ \\
\hline $\begin{array}{l}\text { Lupinus } \\
\text { serecius }\end{array}$ & $\begin{array}{l}E \\
L \\
M\end{array}$ & $\begin{array}{l}5 / 14 \\
5 / 27 \\
5 / 22\end{array}$ & $\begin{array}{l}6 / 24 \\
7 / 5 \\
6 / 30\end{array}$ & $\begin{array}{l}7 / 1 \\
7 / 15 \\
7 / 8\end{array}$ & $\begin{array}{l}7 / 14 \\
7 / 30 \\
7 / 24\end{array}$ & $\begin{array}{l}8 / 1 \\
8 / 12 \\
8 / 5\end{array}$ & $\begin{array}{l}8 / 12 \\
9 / 8 \\
8 / 27\end{array}$ \\
\hline $\begin{array}{l}\text { Oxytropis } \\
\text { sericea }\end{array}$ & $\begin{array}{l}E \\
L \\
N\end{array}$ & $\begin{array}{l}4 / 18 \\
5 / 12 \\
4 / 30\end{array}$ & $\begin{array}{l}6 / 12 \\
7 / 3 \\
6 / 23\end{array}$ & $\begin{array}{l}6 / 25 \\
7 / 10 \\
7 / 3\end{array}$ & $\begin{array}{l}7 / 2 \\
7 / 19 \\
7 / 10\end{array}$ & $\begin{array}{l}8 / 2 \\
8 / 18 \\
8 / 8\end{array}$ & $\begin{array}{l}8 / 11 \\
9 / 30 \\
9 / 5\end{array}$ \\
\hline $\begin{array}{l}\text { Pedicularis } \\
\text { contorta }\end{array}$ & $\begin{array}{l}\mathrm{E} \\
\mathrm{L} \\
\mathrm{M}\end{array}$ & $\begin{array}{l}5 / 1 \\
5 / 21 \\
5 / 10\end{array}$ & $\begin{array}{l}7 / 1 \\
7 / 14 \\
7 / 5\end{array}$ & $\begin{array}{l}7 / 6 \\
7 / 19 \\
7 / 12\end{array}$ & $\begin{array}{l}7 / 13 \\
7 / 27 \\
7 / 21\end{array}$ & $\begin{array}{l}7 / 22 \\
8 / 11 \\
8 / 3\end{array}$ & $\begin{array}{l}8 / 4 \\
9 / 2 \\
8 / 23\end{array}$ \\
\hline $\begin{array}{l}\text { Polygonum } \\
\text { bistortoides }\end{array}$ & $\begin{array}{l}\mathrm{E} \\
\mathrm{L} \\
\mathrm{M}\end{array}$ & $\begin{array}{l}4 / 26 \\
5 / 12 \\
5 / 4\end{array}$ & $\begin{array}{l}6 / 8 \\
6 / 20 \\
6 / 13\end{array}$ & $\begin{array}{l}6 / 15 \\
7 / 1 \\
6 / 22\end{array}$ & $\begin{array}{l}6 / 29 \\
7 / 14 \\
7 / 7\end{array}$ & $\begin{array}{l}7 / 4 \\
7 / 20 \\
7 / 14\end{array}$ & $\begin{array}{l}7 / 20 \\
8 / 2 \\
7 / 27\end{array}$ \\
\hline $\begin{array}{l}\text { Saxifraga } \\
\text { montanensis }\end{array}$ & $\begin{array}{l}E \\
L \\
M\end{array}$ & $\begin{array}{l}4 / 18 \\
5 / 10 \\
4 / 30\end{array}$ & $\begin{array}{l}5 / 12 \\
6 / 2 \\
5 / 21\end{array}$ & $\begin{array}{l}5 / 18 \\
6 / 6 \\
5 / 31\end{array}$ & $\begin{array}{l}6 / 1 \\
6 / 26 \\
6 / 15\end{array}$ & $\begin{array}{l}7 / 7 \\
7 / 27 \\
7 / 16\end{array}$ & $\begin{array}{l}7 / 11 \\
7 / 26 \\
7 / 19\end{array}$ \\
\hline $\begin{array}{l}\text { Zygadenus } \\
\text { venenosus }\end{array}$ & $\begin{array}{l}E \\
L \\
M\end{array}$ & $\begin{array}{l}4 / 18 \\
5 / 10 \\
4 / 28\end{array}$ & $\begin{array}{l}6 / 23 \\
7 / 1 \\
6 / 27\end{array}$ & $\begin{array}{l}7 / 2 \\
7 / 8 \\
7 / 5\end{array}$ & $\begin{array}{l}7 / 7 \\
7 / 16 \\
7 / 12\end{array}$ & $\begin{array}{l}7 / 26 \\
8 / 12 \\
8 / 3\end{array}$ & $\begin{array}{l}6 / 26 \\
8 / 14 \\
7 / 29\end{array}$ \\
\hline
\end{tabular}

${ }^{1}$ Seed set in only 1 of the 5 years. 
Table 2.--Eariiest (E), latest (L), and mean $(M)$ dates of developmental events for grasses on the southwest exposure at 7,100-ft. elevation over a 5-year period.

\begin{tabular}{|c|c|c|c|c|c|c|c|c|}
\hline Species & : Code & $\begin{array}{l}: \text { Active } \\
: \text { growth } \\
: \text { starts }\end{array}$ & $\begin{array}{ll}: & \text { Flower } \\
: & \text { stalks } \\
: & \text { appear } \\
\end{array}$ & $\begin{array}{cc}: & \text { Flowers } \\
: & \text { in } \\
: & \text { bloom } \\
\end{array}$ & $\begin{array}{l}: \\
: \\
:\end{array}$ & $\begin{array}{l}\text { Seed dis- } \\
\text { semination } \\
\text { starts }\end{array}$ & $\begin{array}{l}: \\
: \text { Drying } \\
: \text { begins }\end{array}$ & $\begin{array}{l}: \\
: \text { Plants } \\
: \text { dried }\end{array}$ \\
\hline $\begin{array}{l}\text { Agropyron } \\
\text { spicatum }\end{array}$ & $\begin{array}{l}E \\
L \\
M\end{array}$ & $\begin{array}{l}4 / 25 \\
5 / 15 \\
5 / 4\end{array}$ & $\begin{array}{l}6 / 18 \\
7 / 3 \\
6 / 27\end{array}$ & $\begin{array}{l}7 / 19 \\
7 / 25 \\
7 / 20\end{array}$ & & (1) & $\begin{array}{l}7 / 23 \\
8 / 30 \\
8 / 7\end{array}$ & $\begin{array}{l}9 / 6 \\
9 / 28 \\
9 / 17\end{array}$ \\
\hline $\begin{array}{l}\text { Danthonia } \\
\text { intermedia }\end{array}$ & $\begin{array}{l}E \\
L \\
M\end{array}$ & $\begin{array}{l}5 / 1 \\
5 / 20 \\
5 / 6\end{array}$ & $\begin{array}{l}6 / 22 \\
7 / 2 \\
6 / 27\end{array}$ & $\begin{array}{l}7 / 1 \\
7 / 20 \\
7 / 12\end{array}$ & & $\begin{array}{l}7 / 25 \\
8 / 15 \\
8 / 5\end{array}$ & $\begin{array}{l}7 / 20 \\
8 / 20 \\
7 / 31\end{array}$ & $\begin{array}{c}9 / 8 \\
10 / 12 \\
9 / 22\end{array}$ \\
\hline $\begin{array}{l}\text { Festuca } \\
\quad \text { idahoensis }\end{array}$ & $\begin{array}{l}E \\
L \\
M\end{array}$ & $\begin{array}{l}4 / 25 \\
5 / 16 \\
5 / 4\end{array}$ & $\begin{array}{l}6 / 2 \\
6 / 30 \\
6 / 21\end{array}$ & $\begin{array}{l}7 / 10 \\
7 / 18 \\
7 / 14\end{array}$ & & $\begin{array}{l}7 / 25 \\
8 / 10 \\
8 / 4\end{array}$ & $\begin{array}{l}7 / 23 \\
8 / 20 \\
8 / 3\end{array}$ & $\begin{array}{r}8 / 27 \\
10 / 12 \\
9 / 16\end{array}$ \\
\hline $\begin{array}{l}\text { Koeleria } \\
\quad \text { cristata }\end{array}$ & $\begin{array}{l}E \\
L \\
M\end{array}$ & $\begin{array}{l}4 / 25 \\
5 / 17 \\
5 / 6\end{array}$ & $\begin{array}{l}6 / 7 \\
6 / 28 \\
6 / 22\end{array}$ & $\begin{array}{l}7 / 6 \\
7 / 20 \\
7 / 15\end{array}$ & & $\begin{array}{l}7 / 31 \\
8 / 25 \\
8 / 12\end{array}$ & $\begin{array}{l}7 / 20 \\
8 / 20 \\
8 / 3\end{array}$ & $\begin{array}{l}8 / 21 \\
10 / 7 \\
9 / 11\end{array}$ \\
\hline $\begin{array}{l}\text { Stipa } \\
\quad \text { occidentalis }\end{array}$ & $\begin{array}{l}E \\
L \\
M\end{array}$ & $\begin{array}{l}5 / 1 \\
5 / 18 \\
5 / 7\end{array}$ & $\begin{array}{l}6 / 23 \\
7 / 5 \\
6 / 29\end{array}$ & $\begin{array}{l}7 / 14 \\
7 / 22 \\
7 / 18\end{array}$ & & $\begin{array}{l}7 / 28 \\
8 / 14 \\
8 / 5\end{array}$ & $\begin{array}{l}7 / 30 \\
8 / 20 \\
8 / 8\end{array}$ & $\begin{array}{c}9 / 3 \\
10 / 20 \\
9 / 25\end{array}$ \\
\hline
\end{tabular}

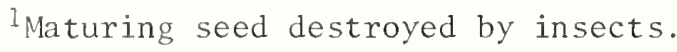

The interval between cessation of flowering and the beginning of seed dissemination differed greatly among forbs. Dezphinium seed began to disseminate an average of only 5 days after flowering, but more than 40 days elapsed before the seed of Anemone, Besseya, and Fritillaria began to fall. These intervals were typically much shorter for late-flowering species (average, 17 days) than for the early bloomers (average 36 days). Usually, grasses began to drop seed between 18 and 28 days after flowering.

Maximum differences between years in date of seed dissemination ranged from 8 to 44 days, according to species; the average was about 20 days regardless of whether the plants were early or late bloomers. Date of seed dissemination for Fritizlaria was least variable from year to year ( 8 days). Species that showed the greatest yearly variation were Agoseris (44 days), Galium (33 days), and Linum (27 days).

Different species dried at widely different times. Those that bloomed early usually dried earlier than those that bloomed late, but there were several exceptions; for example, both Besseya and Anemone bloomed very early (mid-May), but usually did not dry until early and late August, respectively. Conversely, Zygadenus bloomed in early July and was usually dry by the end of that month. Although the grasses usually began to dry in early August, they did not reach the dry stage until approximately the middle of September. Comandra and the grasses were the last species to dry. The leaves of Antennaria apparently remained viable beyond the growing season in which they were formed. Agoseris and Frasera plants that flowered generally dried much sooner than those that remained in a strictly vegetative condition. 
The maximum difference between years in date of drying of various species ranged from 10 to 51 days. Generally, the later a plant flowered, the greater the difference in date of drying between years. The average difference between the earliest and latest drying dates was 19 days for early bloomers, 29 days for midseason bloomers, 33 days for late bloomers, and 39 days for the grasses.

The average length of time that plants remained photosynthetically active ranged from 62 days for Dodecatheon to 147 days for Danthonia (table 3). This active period was considered to extend from the onset of spring growth until the species dried. A general relationship existed between time of flowering and the length of time that plants remained active: early bloomers, 86 days; midseason bloomers, 94 days; late bloomers, 115 days; the early-flowering Anemone, midseasonal Delphinium, and late Lupinus were conspicuous exceptions to this generality (table 3). The grasses remained active for by far the longest period (an average of 137 days). Active growth for all forbs and grasses averaged 108 days.

Table 3.--Shortest, Zongest, and mean periods (in days) of photosynthetic activity over 5 years for plants on the southwest exposure at 7,100 ft.

\begin{tabular}{|c|c|c|c|c|c|c|c|}
\hline Species & Shortest: & Longest: & Mean & Species & Shortest & $:$ Longest: & Mean \\
\hline Dodecatheon conjugens & 50 & 76 & 62 & Campanula rotundifolia & 76 & 127 & 110 \\
\hline Dezphinium bicozor & 71 & 89 & 79 & Geum triflomum & 98 & 131 & 115 \\
\hline Fritizlaria pudica & 66 & 102 & 80 & Anemone patens & 105 & 126 & 116 \\
\hline Saxifraga montanensis & 74 & 93 & 80 & Galium boreale & 111 & 127 & 118 \\
\hline Agoseris glauca & 72 & 98 & 81 & Achizlea mizzefolium & 101 & 150 & 124 \\
\hline Lomatium cous & 77 & 95 & 84 & Koeleria cristata & 96 & 159 & 127 \\
\hline Polygonum bistortoides & 81 & 97 & 84 & Oxutropis sericea & 101 & 152 & 128 \\
\hline Zygadenus venenosus & 69 & 107 & 92 & Comandra umbelzata & 118 & 140 & 132 \\
\hline Cerastium arvense & 83 & 127 & 95 & Agropyron spicatum & 131 & 151 & 135 \\
\hline Besseya wyomingensis & 91 & 99 & 95 & Linum Lewisii & 117 & 146 & 138 \\
\hline Lupinus serecius & 90 & 108 & 99 & Festuca idahoensis & 120 & 169 & 138 \\
\hline Gaizzardia aristata & 86 & 123 & 104 & Stipa occidentalis & 111 & 160 & 139 \\
\hline Pedicularis contorta & 93 & 121 & 106 & Danthonia intermedia & 130 & 164 & 147 \\
\hline Frasera speciosa & 99 & 122 & 110 & & & & \\
\hline
\end{tabular}


Over the 5 years, differences between the shortest and longest periods of active growth averaged 28 days for forbs and 43 days for the grasses. Generally, the period of active growth varied least between years for those species that have short growth periods; it varied most for those species that have long growth periods.

Although most species apparently became dormant for the remainder of the year following drying, several produced new leaves again in the fall. Geum, Saxifraga, Cerastium, and the five grasses shown in figure 2 had this habit. Fall regowth in Geum and Saxifraga was in the form of a small rosette of new leaves within the outer rosette of dried leaves. Holway and Ward (1965) noted this characteristic on Saxifraga rhomboidea growing in alpine tundra. Regrowth of Cerastium was in the form of small leaves in the axils of the dried leaves distributed along the stems. This regrowth usually persisted over winter and appeared to become active again when growth resumed the following spring.

Plant development was delayed on northeast exposures and at the higher elevation (table 4). Some species were affected by these environmental differences much more than others. Depending on species, the start of growth averaged 3 to 12 days later on northeast exposures than on southwest exposures. Oxytropis was an exception; start of growth for this species was delayed an average of 18 days. The general trend was for plants to develop about 1 week later on northeast exposures than on southwest exposures. This difference persisted throughout the growing season. Costello and Price (1939) observed that development on north exposures lagged about 10 days behind that on south exposures on mountain herblands in Utah, whereas Bliss (1956) found only a 2- to 4-day lag on north exposures in the alpine tundra of southern Wyoming.

For individual species, a 1,100-ft. elevational difference delayed beginning of growth from 19 to 37 days (table 4), an average delay of 28 days for all species. Plants at the higher elevation generally developed more rapidly than those at the lower; by the time of flowering, this delay was reduced to an overall average of only 21 days. At the 8,200-ft. elevation, plants dried an average of only 16 days later than at 7,100 ft. The lag in plant development attributed to elevational rise in this study is considerably greater than the 10 days per 1,000 ft. suggested by Hopkins' Bioclimatic Law (Hopkins 1938). It is also greater than the average 12 days per 1,000 ft. observed by Costello and Price (1939) in the mountains of Utah and the average 10-day delay in lilac bloom for each 1,000-ft. elevational rise in Montana (Caprio 1966). This difference in findings might be the result of unusually late snowmelt at the higher elevations on this study area in southwestern Montana. Costello and Price (1939) found that date of snowmelt and onset of growth were closely related, and Holway and Ward (1965) considered snow cover the primary factor influencing phenology of alpine tundra. The response of individual species to elevational differences varied considerably, as Costello and Price (1939) also observed. Growth of Antennaria and Stipa appeared least affected by elevational differences. The beginning of growth for these two species was delayed only about 20 days; flowering of Stipa, 15 days, and of Antennaria, 11 days. Growth of Lomatium appeared to be most affected by elevation; both the start of growth and flowering of this species were delayed an average of 37 days by the 1,100-ft. increase in elevation.

Differences between exposures in the duration of plant growth varied greatly among both species and years. This was also true for differences between elevation. No consistent overall difference in growth duration was apparent between exposures; however, the growth period was generally about 1 week shorter at 8,200-ft. than at 7,100-ft. elevation. For species common to both elevations, the growth period averaged 94 days at 8,200 ft. and 103 days at 7,100 ft. 
Table 4.--Average delay in days (x) over a 5-year period and standard deviation ( $\sigma$ ) of delay in plant development on northeast as compared to southwest exposures, and at 8,200 ft. as compared to 7,100-ft. elevations ${ }^{1}$

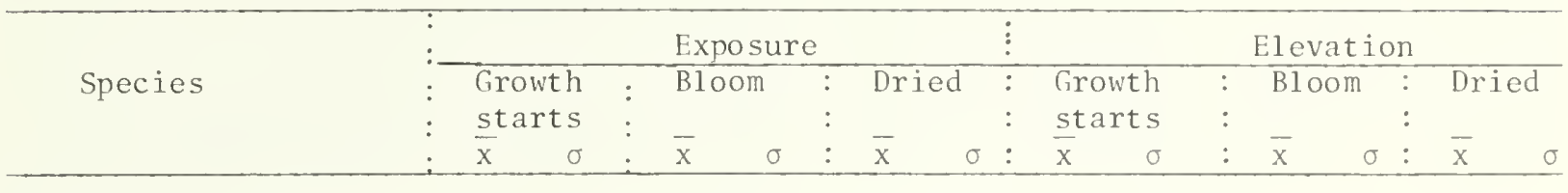

FORBS:

\begin{tabular}{|c|c|c|c|c|c|c|c|c|c|c|c|c|}
\hline Achizlea milzefolium & 7 & 7 & 2 & 5 & 2 & 17 & 32 & 10 & 21 & 11 & 20 & 24 \\
\hline Agoseris glauca & 9 & 4 & 11 & 12 & 21 & 25 & 26 & 9 & 26 & 13 & 34 & 38 \\
\hline Anemone patens & 10 & 2 & 10 & 2 & 5 & 12 & & & & & & \\
\hline Antennaria rosea & 9 & 6 & 5 & 3 & -- & -- & 20 & 6 & 11 & 7 & - - & -- \\
\hline Campanula rotundifolia & 7 & 6 & 2 & 4 & 4 & 10 & & & & & & \\
\hline Cerastium arvense & 3 & 3 & 2 & 4 & 11 & 11 & 27 & 8 & 24 & 7 & 26 & 15 \\
\hline Delphinium bicolor & 5 & 8 & 8 & 5 & 2 & 7 & 29 & 12 & 19 & 12 & 13 & 10 \\
\hline Dodecatheon conjugens & 7 & 5 & 9 & 6 & 17 & 12 & 28 & 9 & 29 & 8 & 25 & 1 \\
\hline Erigeron gracilis & 4 & 2 & 4 & 4 & 1 & 15 & & & & & & \\
\hline Fritizlaria pudica & 6 & 3 & 7 & 7 & 4 & 8 & & & & & & \\
\hline Gailzardia aristata & 8 & 9 & 4 & 1 & 9 & 5 & & & & & & \\
\hline Geum triflorum & 6 & 6 & 6 & 6 & 8 & 12 & 24 & 10 & 22 & 8 & 20 & 0 \\
\hline Linum perenne & 7 & 10 & 3 & 1 & 9 & 5 & & & & & & \\
\hline Lomatium cous & 11 & 4 & 11 & 7 & 5 & 3 & 37 & 10 & 37 & 10 & 14 & 3 \\
\hline Lupinus argenteus & 7 & 6 & 3 & 6 & 1 & 4 & & & & & & \\
\hline Lupinus serecius & 8 & 2 & 5 & 5 & 2 & 5 & & & & & & \\
\hline Myositis syzvatica & & & & & & & 30 & 8 & 20 & 5 & 25 & 10 \\
\hline osytropis serecia & 18 & 5 & 10 & 5 & 2 & 20 & 30 & 13 & 19 & 7 & 15 & 1 \\
\hline Phlox miztiflora & 7 & 4 & -1 & 4 & 7 & 4 & 28 & 5 & 15 & 6 & 8 & 2 \\
\hline Polygonum bistortoides & 7 & 5 & 5 & 5 & 5 & 5 & 29 & 9 & 17 & 6 & 14 & \\
\hline Potentilza diversifolia & 7 & 5 & 5 & 2 & 12 & 12 & & & & & & \\
\hline Saxifraga montanensis & 9 & 5 & 13 & 6 & 7 & 5 & 32 & 5 & 29 & 8 & 4 & 2 \\
\hline Frasera speciosa & & & & & & & 28 & 14 & 19 & 8 & 15 & 4 \\
\hline Zygadenus veneosus & 12 & 10 & 9 & 6 & 15 & 17 & & & & & & \\
\hline
\end{tabular}

GRASSES :

Agropyron spicatum Agropyron caninum Danthonia intermedia Festuea idahoensis Stipa occidentalis

$\begin{array}{rrrr}11 & 4 & 2 & 4 \\ 8 & 1 & 1 & 2 \\ 9 & 9 & <1 & 3 \\ 7 & 5 & 4 & 4 \\ 7 & 6 & 4 & 4\end{array}$

$\begin{array}{rr}5 & 9 \\ 6 & 2 \\ 2 & 3 \\ 9 & 16 \\ 6 & 12\end{array}$

$\begin{array}{rrrrrr}24 & 8 & 13 & 7 & 6 & 7 \\ 27 & 10 & 16 & 5 & 17 & 24 \\ 19 & 7 & 15 & 8 & 8 & 14\end{array}$

${ }^{1}$ Data from lower and upper elevations combined to determine differences between exposures: those from southwest and northeast exposures combined to determine differences between elevations. 


\section{HERBAGE PRODUCTION}

Production of vegetation differed between exposures and fluctuated considerably over the years. Although production was usually greater on the northeast exposures, the magnitude of this difference varied with both elevation and year. Over the 5-year study, the southwest exposure produced only 58 percent as much herbage as the northeast exposure at $7,100-\mathrm{ft}$. elevation, whereas at $8,200 \mathrm{ft}$., it produced 90 percent as much herbage as the northeast exposure. Composition of this vegetation also differed between exposures, but again this difference was not consistent between elevations. At the lower elevation, graminoids formed 58 percent of the total production on the southwest exposure and 26 percent on the northeast exposure. At the upper elevation, these proportions were reversed; graminoids formed 36 percent of the vegetation on the southwest exposure and 58 percent on the northeast exposure.

Herbage production generally fluctuated more between years on northeast than on southwest exposures. Total production on the northeast exposure at 7,100-ft. elevation averaged 1,246 lb./acre with a coefficient of variation (C) of 26 percent; at 8,200-ft. elevation, it averaged $1,127 \mathrm{lb}$ //acre with a $\mathrm{C}$ of 25 percent. However, on southwest exposures, total production at $7,100 \mathrm{ft}$. averaged $724 \mathrm{lb}$./acre with a $\mathrm{C}$ of only 11 percent; at 8,200-ft. elevation, production averaged 1,021 1b./acre with a $\mathrm{C}$ of only 16 percent. The forbs were primarily responsible for this greater variation in total production on the northeast exposures; the $\mathrm{C}$ for forbs averaged 36 percent on the two northeast exposures, and only 17 percent on the two southwest exposures. Variability of graminoid production over the 5 years was about the same on both exposures and at both elevations (C 20 percent).

The amount of variation in production of individual species at the two lower elevation areas is shown in table 5. These data also exemplify production differences between exposures, which were on facing slopes within 200 yards of each other. Relative production of most species fluctuated considerably more between years than did production by vegetation classes. This finding supports Blaisdell's (1958) conclusions that (a) individual species vary greatly in their response to the same environmental factors and (b) increased yield of one can compensate for decreased yield of another and thus dampen oscillations in total production. The great difference between years in relative amounts of minor species (those that contribute little to overall production) is probably the result of sampling inadequacies.

Since 5 years of both production and weather records were available from all four areas, I had an opportunity to relate weather variations to yearly variations in plant production. I assumed at the outset that differences in productive capacity existed between the four areas, irrespective of yearly variations in weather. These area differences were discounted prior to analyses for weather effects. Production for an area 
Table 5.--Herbage production over a 5-year period (1964-1968) on opposite exposures at 7,100-ft. elevation

\begin{tabular}{|c|c|c|c|c|c|c|c|}
\hline \multirow[t]{2}{*}{ Species } & \multicolumn{3}{|c|}{ Southwest exposure } & \multicolumn{4}{|c|}{ Northwest exposure } \\
\hline & : High & : Low & Mean & High : & Low & $:$ & Mean \\
\hline & --- & ---- & -Lb. lacre, & air dry- - & --- & -- & ---- \\
\hline Total vegetation & 817.5 & 604.7 & 723.6 & $1,599.2$ & 723.8 & & $1,246.4$ \\
\hline Total graminoids & 532.3 & 326.2 & 422.7 & 405.7 & 203.4 & & 319.0 \\
\hline Total forbs & 374.3 & 246.4 & 300.9 & $1,294.6$ & 520.4 & & 927.4 \\
\hline \multicolumn{8}{|c|}{ GRAMINOIDS } \\
\hline Agropyron spicatum & 126.6 & 59.5 & 85.7 & 98.8 & 37.6 & & 54.3 \\
\hline Agropyron caninum & 2.6 & 0.1 & 1.2 & 9.8 & 2.3 & & 5.3 \\
\hline Bromus anomalus & & & & 14.8 & 7.0 & & 11.0 \\
\hline Carex fizifolia & 43.0 & 28.1 & 36.1 & 12.2 & 5.8 & & 9.1 \\
\hline Carex stenophyzza & 17.5 & 11.4 & 13.6 & 23.8 & 3.0 & & 11.5 \\
\hline Danthonia intermedia & & & & 58.2 & 5.5 & & 32.9 \\
\hline Festuca idahoensis & 333.3 & 202.0 & 257.3 & 155.2 & 73.4 & & 131.9 \\
\hline Koeleria cristata & 16.2 & 10.5 & 13.8 & 13.4 & 2.8 & & 5.8 \\
\hline Poa spp. & & & & 4.9 & .2 & & 2.2 \\
\hline Stipa occidentalis & 26.3 & 4.8 & 14.8 & 81.0 & 22.3 & & 56.9 \\
\hline \multicolumn{8}{|c|}{ FORBS } \\
\hline Achizlea mizlefolium & 21.9 & 9.3 & 13.7 & 48.6 & 11.5 & & 27.5 \\
\hline Agoseris glauca & 24.6 & 12.9 & 21.1 & 10.4 & 1.3 & & 5.4 \\
\hline Anemone patens & 4.9 & 1.5 & 3.0 & 39.6 & 6.2 & & 24.5 \\
\hline Antennaria rosea & 21.6 & 8.7 & 16.5 & 12.8 & 2.8 & & 9.4 \\
\hline Arenaria congesta & 18.1 & .8 & 8.7 & 69.8 & 2.7 & & 38.9 \\
\hline Astragalus miser & 8.3 & 1.3 & 3.2 & 10.9 & 2.3 & & 5.5 \\
\hline Besseya wyomingensis & 2.3 & .7 & 1.1 & & & & \\
\hline Campanula rotundifolia & 14.1 & 8.3 & 11.2 & 28.2 & 4.3 & & 13.5 \\
\hline Cerastium arvense & 23.2 & 14.8 & 18.3 & 91.6 & 23.2 & & 60.4 \\
\hline Clematis hirsutissima & & & & 18.3 & 4.5 & & 13.2 \\
\hline Comandra umbelzata & 5.3 & 1.8 & 3.0 & & & & \\
\hline Erigeron subtrinervis & 13.7 & 1.4 & 7.8 & 115.7 & 37.8 & & 75.7 \\
\hline Eriogonum umbelzatum & & & & 89.4 & 38.9 & & 53.7 \\
\hline Frasera speciosa & 34.4 & 12.8 & 22.3 & 3.3 & .6 & & 1.7 \\
\hline $\begin{array}{l}\text { Fritillaria pudzea } \\
\text { Gaizzardia aristata }\end{array}$ & 27.0 & 12.2 & 18.4 & 67.5 & 11.6 & & 27.5 \\
\hline Galium boreale & 65.4 & 36.8 & 50.5 & 48.8 & 17.4 & & 27.8 \\
\hline Gentiana affinis & 11.2 & 1.9 & 4.8 & & & & \\
\hline Geranium viscosissimum & & & & 165.8 & 63.8 & & 102.0 \\
\hline Geum triflorum & 12.8 & 5.7 & 8.8 & 139.3 & 45.2 & & 97.3 \\
\hline Gilia congesta & 6.4 & .4 & 2.6 & & & & \\
\hline Linum perenne & 26.7 & 5.8 & 19.6 & 7.8 & 2.3 & & 5.9 \\
\hline Lupinus serecius & & & & 234.6 & 98.1 & & 142.1 \\
\hline Myositis sylvatica & & & & 20.8 & 2.6 & & 12.0 \\
\hline Oxytropis serecia & 5.8 & 1.0 & 3.3 & & & & \\
\hline Pedicularis contorta & 58.9 & 37.4 & 45.5 & & & & \\
\hline Phlox hoodii & 3.2 & .5 & 2.1 & & & & \\
\hline Phzox multiflora & 3.1 & .1 & 1.3 & 105.4 & 74.9 & & 87.6 \\
\hline Polygonum bistortoides & & & & 6.1 & 1.4 & & 3.5 \\
\hline Potentilza gracilis & & & & 20.0 & 4.1 & & 8.3 \\
\hline Taraxacum officinale & 3.1 & .8 & 2.0 & & & & \\
\hline Townsendia mensana & 2.8 & 1.0 & 1.9 & & & & \\
\hline Viola adunca & & & & 112.5 & 40.8 & & 77.5 \\
\hline Zygadenus venenosus & 2.4 & .1 & 1.1 & 9.6 & 1.3 & & 4.7 \\
\hline
\end{tabular}


in each of the 5 years was adjusted by the ratio of individual area mean to the allareas mean; if the interaction between weather and site effects is minimal, this adjustment should effectively discount inherent differences in site productivity. Adjusted productions of total graminoids, of total forbs, and of total vegetation were then evaluated against selected weather factors.

Various expressions of precipitation, air and soil temperatures, solar radiation, wind, and soil moisture status in different months were examined for possible linear relationships to herbage production. Fifty-three such independent variables were examined. From these, I selected the 16 shown in table 6 as possibly being related singly or in combination to the amount of herbage produced every year. The measurement units for these 16 independent variables are:

Soil moisture Precipitation Solar radiation Air temperature

Soil temperature Wind velocity
-- logarithm of ohms electrical resistance;

-- accumulated inches for given period;

- - average cal./cm. $2 /$ day for given period;

-- daily maximum ${ }^{\circ} \mathrm{F}$. averaged for given period;

-- daily minimum ${ }^{\circ} \mathrm{F}$. averaged for given period;

-- degree-hours (the daily average of hours the temperature remained within $5^{\circ} \mathrm{F}$. of the extreme, multiplied by the extreme);

-- same measurement units as air temperature, but measured on a shaded soil surface;

-- average miles per hour for given period.

Few of these variables were independently correlated significantly with the production of either graminoids, forbs, or total vegetation in this limited test. Consequently, the significant positive correlation of production with May precipitation and the negative correlation with the maximum temperatures of the shaded soil surface are particularly conspicuous (table 6). Earlier, I observed that total production on similar mountain grasslands may be related more closely to precipitation falling during the period of most active growth than to precipitation at other times (Mueggler 1967). Smoliak (1956) found production on the short-grass prairies of Alberta to be highly correlated $(r=0.86)$ with May-plus-June precipitation, and significantly negatively correlated $(r=-0.53)$ with seasonal mean temperatures. Failure to verify suspected correlations with soil moisture at the beginning of plant growth might be explained by the small variation in soil moisture between years. Soils on these grasslands usually are near their maximum moisture-holding capacity, down to a depth of $50 \mathrm{~cm}$. at least, at the beginning of plant growth (Mueggler 1971). Ordinarily, this is also true down to $100-\mathrm{cm}$. depths on northeast exposures, but not on southwest exposures. The negative correlation with maximum temperatures of the shaded soil surface and positive correlation with minimum air temperature (degree-hours) suggests that growth of these mountain grassland plants may be favored somewhat by rather cool weather in June and July.

I tested various combinations of independent variables by multiple regression analyses to determine the combination that would best predict herbage yield. A combination of the five following weather measurements gave the highly significant coefficient of multiple determination $\left(R^{2}\right)$ of 0.86 when correlated with total herbage yield: $X_{3}$, May precipitation; $X_{7}$, maximum air temperature $\left({ }^{\circ} \mathrm{F}.\right)$ in July; $X_{8}$, maximum air temperature (degree-hours) in June; $x_{11}$, minimum air temperature (degree-hours) in June; and $x_{14}$, maximum temperature (degree-hours) of shaded soil surface in June. The equation used to estimate total herbage production $\left(\mathrm{Y}_{1}\right)$ has a standard error of estimate of $83 \mathrm{lb} . /$ acre $($ mean production $=1,0301 \mathrm{~b} . /$ acre) :

$$
\hat{Y}_{1}=1,194+169 X_{3}-19.2 X_{7}-2.31 X_{8}+2.40 X_{11}+3.50 X_{14} .
$$


Table 6.--Simple correlation coefficients ( $r$ ) between herbage production and selected weather measurements

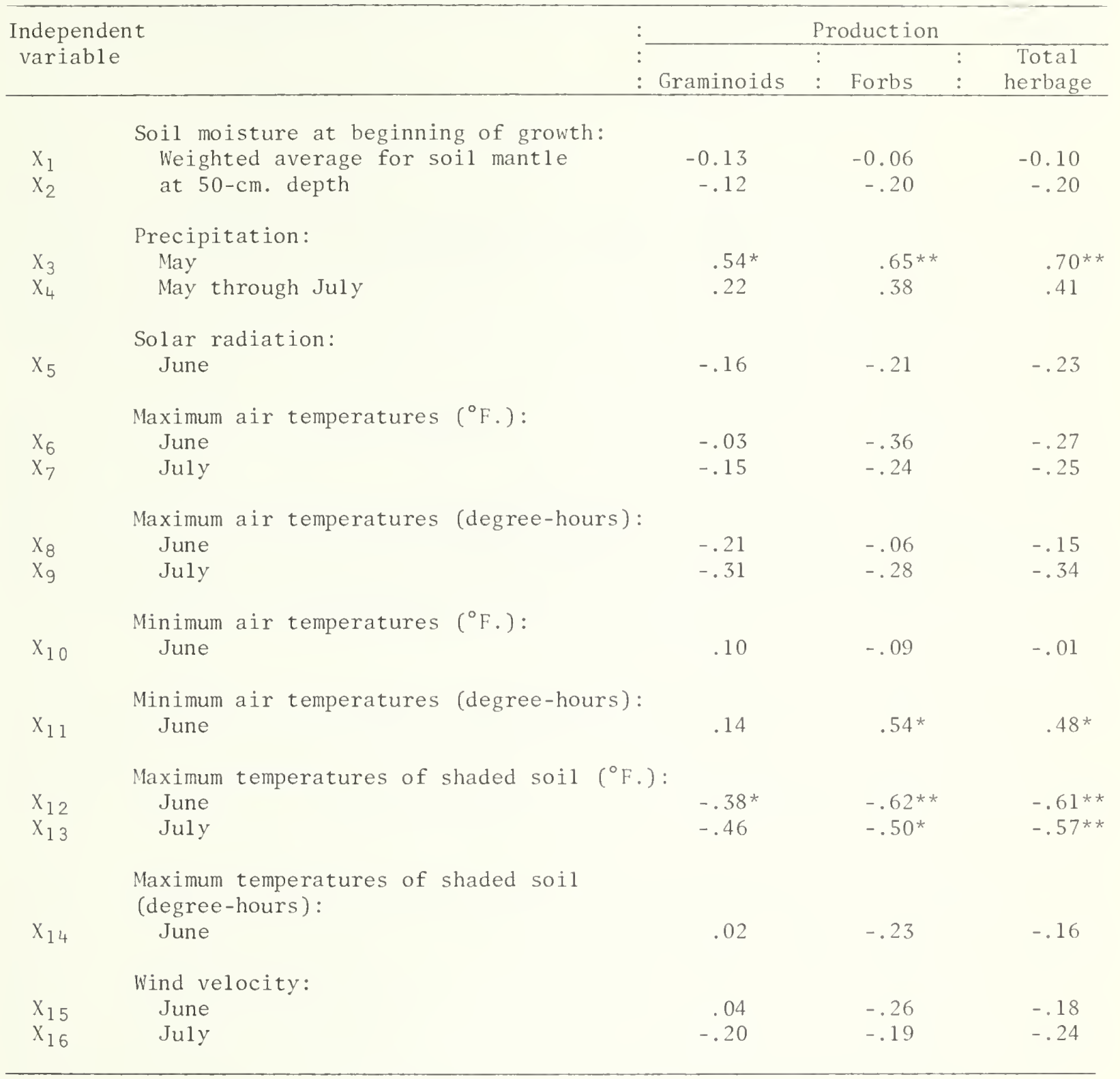

*Significant

**Highly significant 
A different combination of five weather variables gave a highly significant $\mathrm{R}^{2}$ of 0.74 when correlated with total production of forbs. The five independent variables in this case were: $X_{3}$, May precipitation; $X_{5}$, solar radiation in June; $X_{6}$, maximum air temperature $\left({ }^{\circ} \mathrm{F}_{0}\right)$ in June; $\mathrm{X}_{10}$, minimum air temperature ( $\left.{ }^{\circ} \mathrm{F}.\right)$ in June: and $\mathrm{X}_{12}$, maximum temperature ( $\left.{ }^{\circ} \mathrm{F}.\right)$ of shaded soil surface in June. Total forb production $\left(\mathrm{Y}_{2}\right)$ can be estimated with a standard error of estimate of $93 \mathrm{lb} \cdot /$ acre (mean production $=590$ lb./acre) using the following equation:

$$
\hat{Y}_{2}=1,039+95.2 X_{3}+2.29 X_{5}-55.6 X_{6}+70.6 X_{10}-18.2 X_{12} .
$$

Equations developed for predicting total production of graminoids were considerably less precise than those used to predict production of either total vegetation or total forbs. To predict total graminoid production, a combination of four weather measurements was superior to any combination of five tested. The following four independent variables gave a significant $\mathrm{R}^{2}$ value of $0.52: \mathrm{X}_{3}$, May precipitation; $\mathrm{X}_{7}$, maximum air temperature $\left({ }^{\circ} \mathrm{F}.\right)$ in July; $x_{15}$, wind velocity in June; and $x_{16}$, in July. The equation derived for predicting total graminoid production $\left(Y_{3}\right)$ from these four variables has a standard error of estimate of $60 \mathrm{lb} \cdot /$ acre $($ mean production $=439 \mathrm{lb} \cdot / \mathrm{acre})$ :

$$
\hat{Y}_{3}=-415+103 X_{3}+6.91 X_{7}+64.7 X_{15}-41.0 X_{16} .
$$

The correlation coefficients and regression equations examined in this paper are based upon rather tenuous data for meaningful regression analyses. However, they do give some indication of which weather variables may be influential in determining the large yearly variations in herbage production on these mountain grassland ranges. 


\section{MANAGEMENT IMPLICATIONS}

Range managers generally rely upon vegetation and soil readiness indicators to decide when grazing can safely begin in the spring or early summer. Two common indicators of vegetation readiness on mountain grasslands in Montana are DeIphinium bicolor in full bloom and appearance of Festuca idahoensis flower stalks (USDA Forest Serv. 1969). When these indicators were applied to this study, the date of vegetation readiness for grazing was found to differ as much as 26 days during the 5 years. Weather conditions were not extreme during this period; a greater difference will likely occur over a longer time. However, in 2 out of 3 years, vegetation should be ready to graze within 11 days of the average date of readiness, judging from the variability $(\sigma=11.2)$ in date of readiness over the 5 years studied.

Although the lag in plant development attributable to each 1,000-ft. rise in elevation is generally considered to be about 10 days (Hopkins' Bioclimatic Law), the lag apparently can be much greater in some of our mountain areas. In this study, the lag in plant development (and consequently in vegetation readiness for grazing) was 18 days/1,000-ft. rise in elevation. We can also expect a lag of almost 1 week in vegetation readiness on north exposures compared to south exposures.

Knowing how much herbage production is likely to vary from year to year assists the range manager (a) in establishing grazing capacities and (b) in anticipating possible yearly adjustments in stocking. This study shows that herbage production differs much more between years on north exposures than on south exposures. Over this one 5-year period, more than twice as much vegetation was produced on northeast exposures during the high year than during the low year; during this same period, only one-third again as much vegetation was produced on southwest exposures during the high year than the low. Generally, production of forbs varies more between years than production of grasses. In 2 out of 3 years, total herbage production on northeast slopes can be expected to be within 26 percent and that on southwest slopes to be within 14 percent of their respective mean productions.

Regression equations developed in this study for predicting production based upon weather data are primarily of theoretical value. The reruurce manager seldom has access to these types of weather data collected on mountain rangelands. However, both the positive relationship established between production and May precipitation and the negative relationship between production and maximum temperatures in June and July have practical significance. In years when May precipitation is above normal and June and July daytime temperatures are below normal, above-average herbage production can be expected. Conversely, when May precipitation is below normal and June and July temperatures are above normal, below-average herbage production can be expected. 


\section{LITERATURE CITED}

Blaisdell, James P.

1958. Seasonal development and yield of native plants on the Upper Snake River Plains and their relation to certain climatic factors. USDA Tech. Bull. $1190,68 \mathrm{p}$.

Bliss, L. C.

1956. A comparison of plant development in microenvironments of arctic and alpine tundras. Ecol. Monogr. 26(4):303-337.

Caprio, J. M.

1966. Pattern of plant development in the western United States. Mont. Agr. Exp. Sta. Bull. 607, 42 p.

Costello, David F., and Raymond Price

1939. Weather and plant development data as determinants of grazing periods on mountain range. USDA Tech. Bull. 686, $31 \mathrm{p}$.

Holway, J. G., and R. T. Ward

1965. Phenology of alpine plants in northern Colorado. Ecology 46(1): 73-83.

Hopkins, A. D.

1938. Bioclimatics--a science of life and climatic relations. USDA Misc. Publication 280, 188 p.

Hutchings, Selar S., and Jack E. Schmautz

1969. A field test of the relative-weight-estimate method for determining herbage production. J. Range Manage. 22(6): 408-411.

Mueggler, W. F.

1967. Response of mountain grassland vegetation to clipping in southwestern Montana. Ecology 48(6): 942-949.

1971. Weather variations on a mountain grassland in southwestern Montana. USDA Forest Service Res. Pap. INT-99,"25 p.".

Smoliak, S.

1956. Influence of climatic conditions on forage production of shortgrass

rangeland. J. Range Manage. 9(2): 89-91.

USDA Forest Service

1969. Range environmental analysis handbook. FSH 2209.21, R-1. 


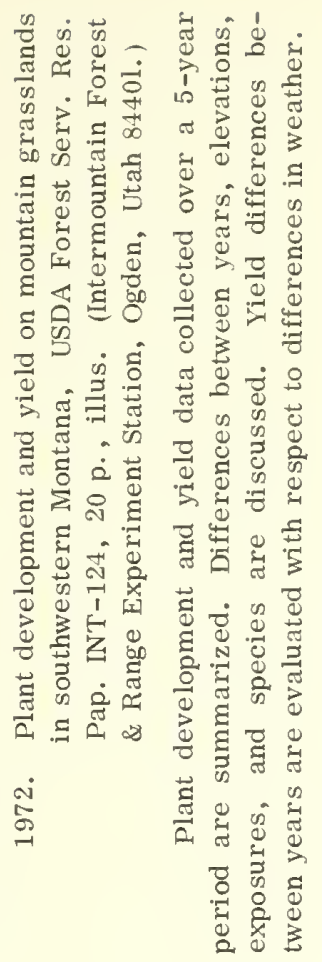

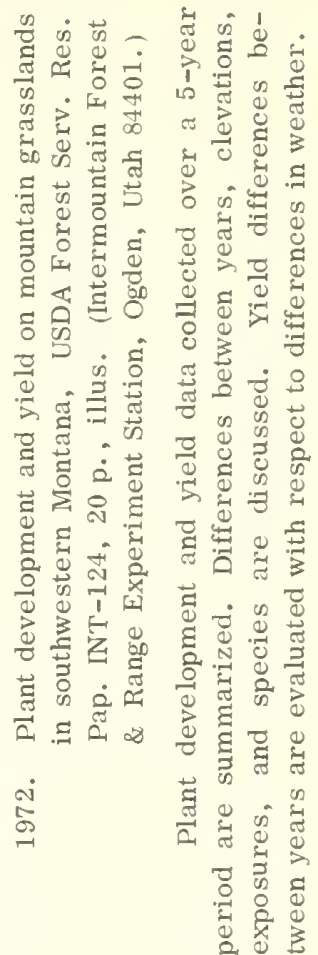

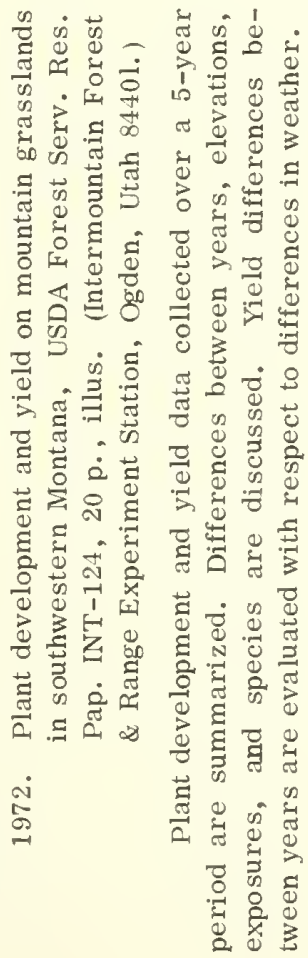

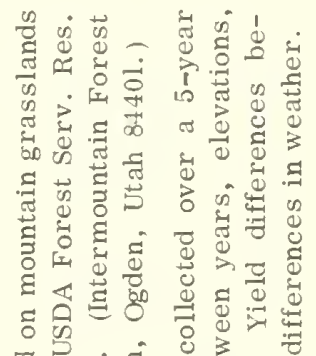

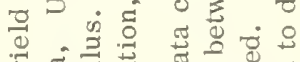

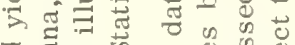

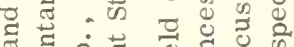

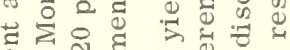

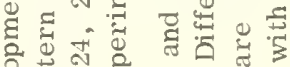

届可则

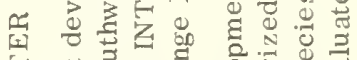

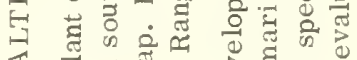

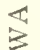

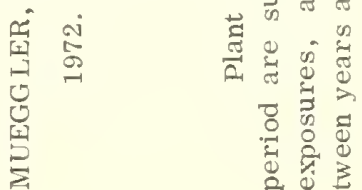



Headquarters for the Intermountain Forest and Range Experiment Station are in Ogden, Utah. Field Research Work Units are maintained in:

Boise, Idaho

Bozeman, Montana (in cooperation with Montana State University)

Logan, Utah (in cooperation with Utah State University)

Missoula, Montana (in cooperation with University of Montana)

Moscow, Idaho (in cooperation with the University of Idaho)

Provo, Utah (in cooperation with Brigham Young University) 


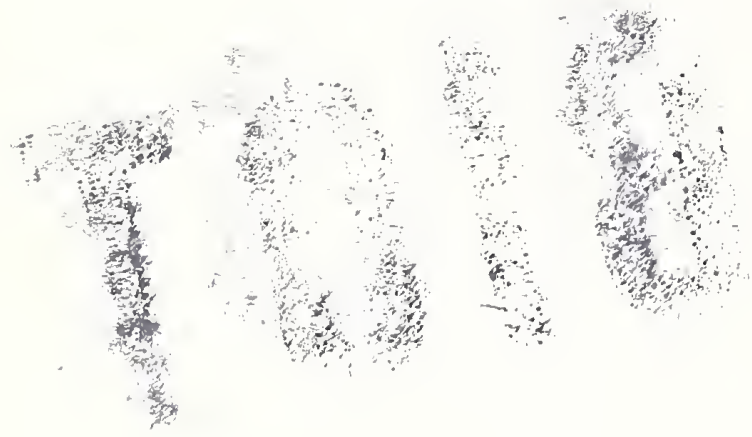

